\title{
Une condition de Dini-Lipschitz dans la théorie du potentiel
}

\author{
par W. KLEINER (Kraków)
}

\$ 1. Introduction. Considérons les mesures positives $\mu$ portées par un ensemble plan compact $\Gamma$. On sait que si l'énérgie d'une telle mesure est petite, sa masse totale l'est aussi. Plus précisément,

$$
\mu(\Gamma) \leqslant c_{\mathbf{0}}\|\mu\|,
$$

$\|\mu\|$ désignant la racine carrée de l'énergie (7); la constante $c_{0}>0$ ne dépend que de $\Gamma$. La démonstration est facile: on cherche le minimum $1 / c_{0}$ de $\|\mu\|$ dans la classe des mesures de masse totale 1: $\mu(\Gamma)=1$. Pour une mesure $\mu$ quelconque sur $\Gamma$, on a alors $1 / c_{0} \leqslant\|\mu / m\|$ où $m=\mu(\Gamma)$, c'est-à-dire (1). $\Pi$ est essentiel ici que les mesures positives forment un cône: si $\mu$ est une d'elles, $t \mu(t>0)$ l'est aussi.

Nous nous proposons d'étendre l'inégalité (1) - ou au moins une inégalité semblable - aux mesures générales $\mu=\mu^{+}-\mu^{-}\left(\mu^{+}, \mu^{-}-\right.$mesures positives). Il est clair que, dans ce cas général, le premier membre de l'inégalité (1) doit changer de forme, car dans le cas le plus important où $\mu(\Gamma)=0$ elle deviendrait triviale. D'autre part, l'idée naturelle de remplacer $\mu(\Gamma)$ par la masse totale absolue $|\mu|(\Gamma)$ (voir (11)) est aussi à rejeter, car en plaçant les masses (charges) positives sur les intervalles $(2 k / n,(2 k+1) / n)$ et les masses négatives sur les intervalles $((2 k+1) / n$, $(2 k+2) / n)(k==0,1, \ldots, n-1)$, on peut (pour un $n$ suffisamment grand) rendre $\|\mu\|$ aussi petite et $|\mu|(\Gamma)$ aussi grand que l'on veut. Donc une norme nouvelle est à appliquer, ce qui nous conduit, entre autres raisons, à de sérieuses restrictions sur la forme du support: $\Gamma$ doit être une courbe, ou un arc, ̀̀ tangente continue. Or, nous posons

$$
[\mu]=\sup |\mu(L)|
$$

où la borne supérieure est prise par rapport à tous les arcs partiels $L$ de $I$.

Mais, l'inégalité de Lipschitz (1) ne s'étend pas aux mesures générales, même dans ces conditions. On peut seulement établir une condition de Dini-Lipschitz (th. 6)

$$
[\mu] \leqslant e^{\prime}\|\mu\| \log \frac{1}{\|\mu\|}
$$


si $\mu$ est de densité bornée; dans le cas plus général il faut se contenter d'une inégalité de Hölder

$$
[\mu] \leqslant c^{\prime \prime}\|\mu\|^{1 / 2} .
$$

Toutes les considérations exigent une majoration par une mesure fixée; nous n'avons plus un cône de mesures, c'est pourquoi la démonstration est pénible et le résultat n'a pas l'élégante simplicité de (1).

La condition sur $\Gamma$ peut être un peu affaiblie; mais la présence d'un point de rebroussement peut produire un condensateur, pour lequel (3) ni (4) ne sont plus valables.

En s'appuyant sur l'inégalité (3) ou (4), on peut obtenir une limitation pour la différence des potentiels (5) $U^{\mu}-U^{*}$ moyennant l'inégalité du théorème 8:

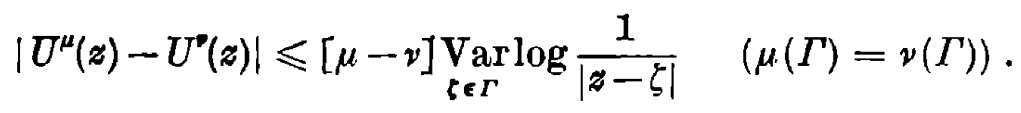

\$ 2. Soit $\Gamma$ une courbe simple fermée, ou bien un are simple, de classe $C^{1}$ (a tangente continue) sur le plan complexe, de longueur $|\Gamma|<1$, $A$ la classe de ses ares partiels, $S$ - celle des ensembles de Borel dans $\Gamma$. Nous appelons mesure toute fonction $\mu$ totalement additive sur $S$. Son potentiel $U^{\mu}$ est introduit par la formule

$$
U^{\mu}(z)=\int \log \frac{1}{|z-\zeta|} d \mu(\zeta)
$$

et le produit intérieur (l'énergie mutuelle) de deux mesures est défini comme il suit:

$$
(\mu, v)=\int U^{\mu}(z) d v(z)
$$

Ce produit est une forme linéaire en $\mu$ et $\nu$ séparément. Nous nous bornons aux mesures pour lesquelles l'intégrale d'énergie $\|\mu\|^{2}=(\mu, \mu)$ est finie. En particulier, ces mesures sont continues. En vertu du théorème de Fubini on a alors $(\mu, \nu)=(\nu, \mu)$; et comme $(\mu, \mu)>0$, sauf pour $\mu \equiv 0$, ce qui suit d'un théorème de [1], nos mesures forment un espace préhilbertien réel avec la norme

$$
\|\mu\|=(\mu, \mu)^{1 / 2} .
$$

Néanmoins, nous n'utiliserons pas la topologie induite par cette norme, mais la topologie de la convergence simple [1]. Cette topologie, dite topologie vague, s'introduit, de façon la plus convenable, par la définition suivante [2]:

(8) $\mu_{n} \rightarrow \mu$ si $\int f d \mu_{n} \rightarrow \mid f d \mu$ pour toute fonction $f$ continue sur $\Gamma$. 
Nous écrirons

$$
\begin{array}{cl}
\mu_{1} \leqslant \mu_{2} & \text { si } \quad \mu_{1}(E) \leqslant \mu_{2}(E) \quad(E \in S), \\
\mu=a_{1} \mu_{1}+a_{2} \mu_{2} \quad \text { si } \quad \mu(E)=a_{1} \mu_{1}(E)+a_{2} \mu_{2}(E) \quad(E \in S) . \\
\text { mesure } \geqslant 0 \text { est dite } \text { positive. }
\end{array}
$$

Pour une mesure $\mu$, soient $\mu^{+}$et $\mu^{-}$ses variations, supérieure et inférieure respectivement; $\mu=\mu^{+}-\mu^{-}$. Nous posons

$$
|\mu|=\mu^{+}+\mu^{-} \text {; }
$$

$|\mu|(\Gamma)$ est dite variation totale de $\mu$.

Soit encore une autre norme

$$
[\mu]=\sup _{L \in A}|\mu(L)| \text {. }
$$

Observons qu'il y a un arc $L^{*}$ pour lequel $\left|\mu\left(L^{*}\right)\right|=[\mu]$, ce qui résulte de la continuité de $\mu$. Notre but est d'étudier les relations entre ces deux normes dans certains sous-ensembles de l'espace des mesures.

§ 3. Nous commençons par la théorie de l'équilibre. Soit $v$ une mesure positive, s'annulant en dehors de $\Gamma$, de potentiel continu sur $\Gamma$, et $K=K(m, v)$ la classe des mesures $\sigma$ sur $\Gamma$ telles que

$$
|\sigma| \leqslant v, \quad[\sigma] \geqslant m, \quad \sigma(\Gamma)=0 .
$$

$K$ est compact. Soit, en effet, $\sigma_{n} \in K, \sigma_{n}\left(L_{n}\right)=\left[\sigma_{n}\right], L_{n} \in A$. Coinme les rariations satisfont $\grave{a}$ la condition $\left|\sigma_{n}\right|(\Gamma) \leqslant|\nu|(\Gamma)$, le théorème de Helly nous permet d'en tirer une suite convergente $\sigma_{n_{k}} \rightarrow \sigma$; nous pouvons obtenir aussi $L_{n_{k}} \rightarrow L_{0},\left[\sigma_{n_{k}}\right] \rightarrow m^{*} \geqslant m$. Posons $L_{n_{k}}=L_{0} \pm A_{k} ; A_{k}$ est un arc ou la réunion de deux ares (le signe -.- ou -.- est pris convenablement pour chacun d'eux séparement). Alors

$$
\left|\sigma_{n_{k}}\left(L_{0} \pm A_{k}\right)-\sigma\left(L_{0}\right)\right| \leqslant\left|\sigma_{n_{k}}\left(L_{0}\right) \cdots \sigma\left(L_{0}\right)\right|+v\left(A_{k}\right) \rightarrow 0,
$$

d'où $\sigma\left(L_{0}\right)=m^{*} \geqslant m$. De même $|\sigma| \leqslant \nu$ et $\sigma(\Gamma)=0$, donc $\sigma \in K$.

La fonctionnelle $\|\sigma\|$ est continue dans $K$; cela résulte d'un lemme dont la généralisation nous semble connue. Nous en donnons, néanmoins, une démonstration à cause de sa simplicité.

LEMME 1. Si $\sigma_{n} \rightarrow \sigma,\left|\sigma_{n}\right| \leqslant \nu$ et $\|\nu\|<\infty$, alois $\left\|\sigma_{n}\right\| \rightarrow\|\sigma\|$.

Démonstration. Soit $I_{1}(r)$ une fonction égale à $\log 1 / r$ pour $0 \leqslant r \leqslant \frac{1}{2} \delta$, linéaire pour $\frac{1}{2} \delta \leqslant r \leqslant \delta$, nulle pour $\delta \leqslant r \leqslant 1$, et $L_{2}(r)$ $=\log 1 / r-L_{1}(r)$. On a $\left\|\sigma_{n}\right\|^{2}-\|\sigma\|^{2}=I_{1}-I_{2}, \quad I_{k}=\int L_{k}(|z-\zeta|) d\left(\sigma_{n} \otimes \sigma_{n}-\right.$ $-\sigma \otimes \sigma)(k=1,2)$; la signification de $\otimes([2]$, III, 5) est manifeste.

Un $\varepsilon>0$ étant donné, il existe un $\delta>0$ tel que $\int L_{1}(|z-\zeta|) d \nu$ $\otimes \nu<\varepsilon\left(\operatorname{car}\|v\|^{2}<\infty\right.$ et $\left.0 \leqslant L_{1}(r) \leqslant \log 1 / r\right)$; mais $\left|\sigma_{n} \otimes \sigma_{n}\right|,|\sigma \otimes \sigma| \leqslant \nu \otimes v$, et nous avons alor's $\left|I_{1}\right|<2 \varepsilon$. Fixons ce $\delta$. On a $\sigma_{n} \otimes \sigma_{n} \rightarrow \sigma \otimes \sigma$ ([2], III, 5, 
prop. 4) et $L_{2}(|z-\zeta|)$ est continue sur $\Gamma \times \Gamma$, pour les $n$ suffisamment grands on a done $\left|I_{1}+I_{2}\right| \leqslant\left|I_{1}\right|+\left|I_{2}\right|<2 \varepsilon+\varepsilon$, et le lemme est établi.

Le théorème d'équilibre en est maintenant une conséquence tout à fait naturelle.

THÉORÈME 1. Il existe dans $K$ une mesure minimale $\alpha$, telle que

$$
\|a\| \leqslant\|\sigma\| \quad(\sigma \in K) .
$$

Démonstration. $K$ est compact et $\|\sigma\|$ continue dans $K$.

Evidemment, le théorème reste valable lorsqu'on y remplace la classe $K$ par une sous-classe fermée quelconque $Q$; nous en ferons usage.

Examinons maintenant la distribution de masse et le potentiel d'équilibre.

THÉonìme 2 (sur la neutralisation des charges). On peut partager $\Gamma$ en deux arcs $L^{+}, L^{-}$tels que $\alpha \geqslant 0$ dans $L^{+}, \alpha \leqslant 0$ dans $L^{-}$.

Démonstration. Il existe un arc $L$ pour lequel $\alpha(L)=[\alpha]$. Nous posons $L^{+}=L, L^{-}=\overline{\Gamma-L^{+}}$. Soit $Q$ la classe des mesures $\sigma$ de $K$, positives dans $L^{+}$, négatives dans $L^{-}$pour lesquelles $\sigma\left(L^{+}\right)=m$. La classe $Q$ est fermée, il existe donc dans $Q$ une mesure minimale $\beta:\|\beta\| \leqslant\|\sigma\|$ pour $\sigma \in Q .\left(U^{\beta}(\infty)=0\right.$, car $\beta(\Gamma)=0$.) Soit

$$
V=\sup _{z \in \Gamma} U^{\beta}(z), \quad v=\inf _{z \in \Gamma} U^{\beta}(z) .
$$

Ces quantités sont finies en vertu du lemme 2. Il nous faut s'appuyer sur le théorème:

THÉORÈnE 3 (de saturation). $U^{\beta}(z)=V$ sur $L^{+}$, sauf pour un ensemble $A \subset L^{+}$, pour lequel $\beta(A)=v(A)$. De même, $U^{\beta}(z)=v$ sur $L^{-}$, sauf pour un ensemble $B \subset L^{-}$tel que $\beta(B)=-v(B)$.

Supposons ce théorème établi. Soit $a=\beta+\tau$. On a $\tau \leqslant 0$ dans $A$, $\tau \geqslant 0$ dans $B, \tau\left(L^{+}\right)=\tau\left(L^{-}\right)=0$ et l'intégration de $U^{\beta} d \tau$ nous donne:

$$
\int_{A} \geqslant V \tau(A), \quad \int_{L^{+}-A}=V \tau\left(L^{+}-A\right), \quad \int_{B} \geqslant v \tau(B), \quad \int_{L^{-}-B}=v \tau\left(L^{-}-B\right) .
$$

Comme $a, \beta \in K$, on a $\tau(\Gamma)=0$, alors la somme de ces intégrales est positive: $(\beta, \tau) \geqslant 0$, donc

$$
\|\alpha\|^{2}=\|\beta\|^{2}+2(\beta, \tau)+\|\tau\|^{2}>\|\beta\|^{2}
$$

sauf pour $\tau \equiv 0$. Mais, $\beta \in K$, d'où $\|\alpha\| \leqslant\|\beta\|$. Donc $\tau \equiv \mathbf{0}$, et $\alpha \equiv \beta$.

Dans la démonstration du théorème 3, nous aurons recours au simple lemme suivant:

LEMME 2. Si $|\sigma| \leqslant \mu$ et $U^{\mu}$ est continu au point $z_{0}$, $U^{\sigma}$ l'est aussi.

Démonstration (cf. [4], th. $\mathbf{A}_{2}$ ). $\pm U^{\sigma}=U^{\mu \pm \sigma}-U^{\mu} ; \mu \pm \sigma \geqslant 0$, donc $U^{\sigma}$ et $-U^{\sigma}$ sont semi-continus inférieurement au point $z_{0}$, [5]. 
Démonstration $d u$ théorème 3. Supposons $\nu(A)-\beta(A)>0$, et soit $A_{s}$ l'ensemble des points de $L^{+}$pour lesquels $U^{\beta} \leqslant V-\varepsilon$. Pour $\varepsilon>0$ suffisamment petit on a $a=\nu\left(A_{s}\right)-\beta\left(A_{s}\right)>0, U^{\beta}$ est continu par le lemme $2, A_{\varepsilon}$ est donc fermé. On a $b=\beta\left(L^{+}-A_{s}\right)>0$; sinon, $U^{\beta}$ serait une fonction sousharmonique en dehors de $A_{\varepsilon}$ et continue partout; on aurait alors $U^{\beta} \leqslant \nabla-\varepsilon$ et $V$ ne serait pas son maximum. Posons

$$
\begin{array}{llll}
\tau(E)=a \beta(E) & \text { si } & E \subset L^{+}-A_{\varepsilon}, & E \in S, \\
\tau(E)=-b(\nu-\beta)(E) & \text { si } & E \subset A_{\varepsilon}, & E \in S ;
\end{array}
$$

alors $\tau\left(L^{+}\right)=0$. On a

où

$$
\|\beta-t \tau\|=\|\beta\|^{2}-2 t(\beta, \tau)+t^{2}\|\tau\|^{2},
$$

$$
(\beta, \tau)=\int_{A_{\varepsilon}} U^{\beta} d \tau+\int_{L^{+}-A_{\varepsilon}} U^{\beta} d \tau>(V-\varepsilon) \tau\left(A_{\varepsilon}\right)+(V-\varepsilon) \tau\left(L^{+}-A_{\varepsilon}\right)=0 ;
$$

pour $t>0$ suffisamment petit $\beta-t \tau \in Q$ et son énergie est plus petite que celle de $\beta$; donc, $\beta$ ne peut être minimale.

Remarque 1. Les supports de $a=\beta$ et de $v$ sont identiques.

Il suffit de le démontrer pour les parties contenues dans $L^{+}$et $L^{-}$ séparément. Si $\beta\left(L^{+}\right)=v\left(L^{+}\right)$, il n'y a rien à prouver. Soit $\beta\left(L^{+}\right)<v\left(L^{+}\right)$, est soit $z_{0} \in L^{+}$un point du support de $v$ extérieur au support de $\beta$. I existe alors un are $L^{\prime} \subset L^{+}$contenant $z_{0}$ tel que $v\left(L^{\prime}\right)>0, \beta\left(L^{\prime}\right)=0$. $U^{\beta}$ étant continu et harmonique sur $L^{\prime}$ (et non constant dans son voisinage) nous pouvons diminuer $L^{\prime}$ de façon que pour un $\varepsilon>0$ on ait $U^{\beta} \leqslant V-\varepsilon$ sur cet arc et que $v\left(L^{\prime}\right)=a$ reste positif. De même que dans la démonstration du théorème 3 on a $b=\beta\left(L^{+}-A_{\varepsilon}\right)>0$. Nous posons

$$
\begin{array}{llll}
\tau(E)=-b \nu(E) & \text { si } & E \subset L^{\prime}, & E \in S, \\
\tau(E)=a \beta(E) & \text { si } & E \subset L^{+}-A_{\varepsilon}, & E \in S
\end{array}
$$

et la démonstration s'achève comme la précédente.

THÉORìme 4. Si $a_{1}, a_{2}$ sont deux mesures minimales dans $K$ et le support de $\alpha_{1}^{+}$est identique à celui de $a_{2}^{+}$, ces mesures sont identiques.

Démonstration. On peut poser $L_{1}^{+}=L_{2}^{+}$et appliquer la seconde partie de la démonstration du théorème 2.

\$4. Un lemme de caractère géométrique nous sera indispensable.

Nous mesurons les angles entre deux droites au sens absolu: un angle est alors compris entre 0 et $\pi / 2$. Nous dirons qu'un arc $L$ est incliné sur la droite $L$ d'un angle $\geqslant \varepsilon$, si toutes les tangentes à $L$ satisfont à cotte condition.

Pour notre courbe (ou notre arc) $\Gamma \epsilon C^{1}$ fixons un $r>0$, tel que pour tout point $z_{0} \in \Gamma$ la partie de $\Gamma$ contenue dans un cercle $C_{r}=\{z$ : 
$\left.\left|z-z_{0}\right| \leqslant r\right\}$ se compose d'un arc, incliné d'un angle $\geqslant \pi / 3$ sur la droite $l_{0}$ normale à $\Gamma$ au point $z_{0}$.

LEMME 3. Pour une courbe (ou un arc) $\Gamma$ à tangente continue, on peut donner des constantes $\vartheta>0, \varepsilon>0, N$, jouissant des propriétés suivantes:

Un point $z_{a} \in \Gamma$ étant donné,

a) il existe une droite $l$, passant par $z_{0}$ et telle que

(14) $\Gamma$ est la réunion des arcs simples $L_{1}, \ldots, L_{n}(n \leqslant N)$, inclinés sur $l$ d'un angle $\geqslant \varepsilon$, et d'un ensemble $\Gamma^{\prime}$, dont la distance à $l$ est $\varrho\left(\Gamma^{\prime}, l\right) \geqslant \vartheta$;

b) il existe deux droites $l_{1}, l_{2}$, orthogonales $a$ la normale $l_{0}$, satisfaisant à la condition (14), et telles que le point $z_{0}$ soit situé entre elles à une distance $\varrho\left(z_{0}, l_{i}\right) \in(r / 2, r)(i=1,2)$ (fig. 1).

Observons que les droites $l_{1}$ et $l_{1}$ ne coupent pas $\Gamma$ à l'intérieur de $C_{r}$.

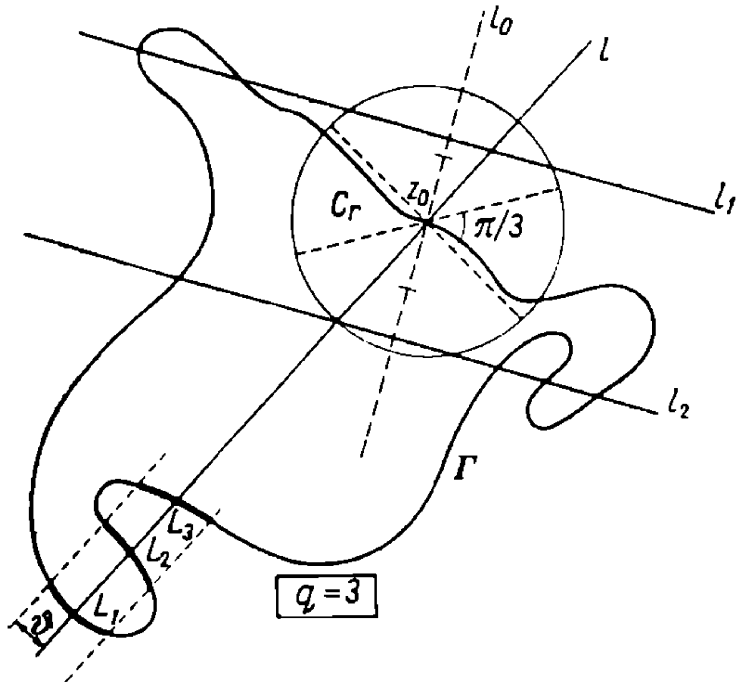

Fig. 1

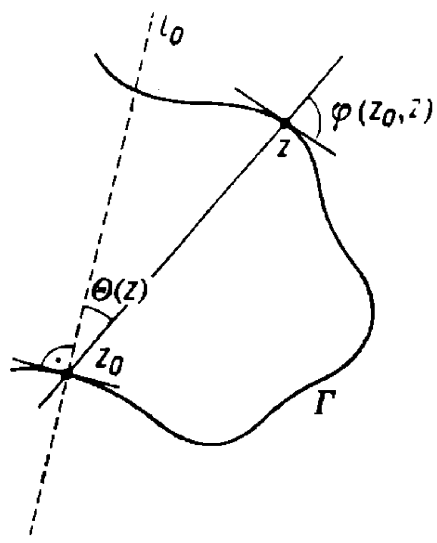

Fig. 2

Démonstration. Soit $\varphi=\varphi\left(z_{0}, z\right)$ l'angle du rayon $z_{0} z$ avec la tangente à $\Gamma$ au point $z$, et $\theta(z)$ l'angle formé par le même rayon et la droite $l_{0}$ (fig. 2). $\varphi$ et $\theta$ sont uniformément continues dans les ensembles $\Gamma \times \Gamma$ et $\Gamma$ respectivement. Désignons par $\Gamma_{\varepsilon}$ l'ensemble des points $z \in \Gamma$ où $\varphi\left(z_{0}, z\right)<\varepsilon$. L'ensemble des valeurs de $\theta(z)$ sul $\Gamma_{2 \varepsilon}$ :

$$
E_{1}=\theta\left(\Gamma_{2 \varepsilon}\right)=\left\{\theta: \theta=\theta(z), z \epsilon \Gamma_{2 \varepsilon}\right\}
$$

est de mesure linéaire $\left|E_{1}\right|<\left|\Gamma_{2 \varepsilon}\right| R^{-1} \sin 2 \varepsilon<2 \varepsilon|\Gamma| R^{-1} \quad(R=$ dianètre de $\Gamma$ ). Nous fixons $\varepsilon=\pi R / 16|\Gamma|$; donc, $\left|E_{1}\right|<\pi / 8$. Fixons encore un $\delta>0$ tel que, pour $\left|z_{1}-z_{2}\right|<\delta, z_{0} \in \Gamma$,

$$
\left.\mid \varphi\left(z_{0}, z_{1}\right)-\infty i z_{0}, z_{2}\right)|<\varepsilon, \quad| \theta\left(z_{1}\right)-\theta\left(z_{2}\right) \mid<\varepsilon,
$$

et que l'angle formé par les tangentes à $\Gamma$ aux points $z_{1}$ et $z_{2}$ soit aussi $<\varepsilon$. 
L'ensemble $\Gamma_{\text {c }}$ est la réunion d'une famille dénombrable d'arcs ouverts dans $I$. Soit $L$ un de ces arcs, extrémités comprises. Choisissons deux points $z_{1}, z_{2} \in L$ pour lesquels $\theta$ prend ses valeurs extrémales: $\theta\left(z_{1}\right)$ $\leqslant \theta(z) \leqslant \theta\left(z_{2}\right) \quad(z \in L)$. Le point $z_{1}$ est une des extrémités de $L$ (alors $\varphi\left(z_{0}, z_{1}\right)=\varepsilon$ ), ou bien un point intérieur de $L$. Dans ce dernier cas nous l'appelons point critique; on y a $\varphi\left(z_{0}, z_{1}\right)=0$, d'où $|L| \geqslant 2 \delta$. D'une manière analogue, $z_{2}$ peut être une extrémité de $L$ ou bien un point critique. Il n'y a alors que $n \leqslant N_{0}=|\Gamma| / 2 \delta$ arcs composants de $\Gamma_{\varepsilon}$ qui contiennent des points critiques, chaque arc en contenant deux au plus; désignons les valeurs de $\theta$ qui correspondent aux points critiques par $\theta_{1}, \ldots, \theta_{k}\left(k \leqslant 2 n \leqslant 2 N_{0}\right)$. Soit $\vartheta^{\prime}=\delta \pi / 8|\Gamma|$, et

$$
E_{2}=\bigcup\left\langle\theta_{i}-\vartheta^{\prime}, \theta_{i} \vartheta^{\prime}\right\rangle, \quad \boldsymbol{E}=E_{1} \cup E_{2} .
$$

On a $|\boldsymbol{E}|<\pi / 4$, il existe donc dans l'intervalle $\langle 0, \pi / 4\rangle$ un $\theta_{0} \xi E$. Soit $l$ une droite passant par $z_{0}$ et inclinée d'un angle $\theta_{0}$ sur $l_{0}$. Fixons un point d'intersection de $l$ avec $\Gamma: \zeta \in l \cap \Gamma$; on a $\varphi\left(z_{0}, \zeta\right) \geqslant 2 \varepsilon$. Soit $L(\zeta)$ le plus grand arc contenant $\zeta$ et incliné sur $l$ d'un angle $\geqslant \varepsilon$; les tangentes à $L$ au point $\zeta$ et à l'une des extrémités (et à l'autre) forment un angle $\geqslant \varepsilon$, donc $|L(\zeta)| \geqslant 2 \delta$. Si $\zeta^{\prime}$ est un autre point de $l \cap \Gamma, L\left(\zeta^{\prime}\right)$ et $L(\zeta)$ sont disjoints. Il n'y a alors que $q \leqslant N_{0}$ points d'intersection $\zeta_{1}, \ldots, \zeta_{q} \in l \cap$ $\cap\left(\Gamma-C_{r}\right)\left(C_{r}\right.$ défini an début de ce paragraphe). Posons $L_{j}=L\left(\zeta_{j}\right)$, $I_{q+1}=\Gamma \cap C_{r}, \Gamma^{\prime}=\overline{\Gamma-\bar{\bigcup} L_{j}}$. Il existe un $z^{\prime} \in \Gamma^{\prime}$ tel que

$$
\left|\theta\left(z^{\prime}\right)-\theta_{0}\right| \leqslant\left|\theta(z)-\theta_{0}\right| \quad\left(z \in \Gamma^{\prime}\right) \text {. }
$$

Ise point $z^{\prime}$ peut être:

1) une extrémité de $L_{q+1}$; alors $\left|\theta\left(z^{\prime}\right)-\theta_{0}\right| \geqslant \pi / 12=\vartheta_{1}$,

2) une extrémité d'un arc $L_{j}(j \leqslant q)$; alors $\left|\theta\left(z^{\prime}\right)-\theta_{0}\right|>\delta \sin \varepsilon j R=\vartheta_{2}$,

3) un point critique; alors $\left|\theta\left(z^{\prime}\right)-\theta_{0}\right|=\left|\theta_{j}-\theta_{0}\right|>\vartheta^{\prime}$.

Done, pour $\vartheta_{0}=\min \left(\vartheta_{1}, \vartheta_{2}, \vartheta^{\prime}\right)$

$$
\left|\theta(z)-\theta_{0}\right| \geqslant \vartheta_{0} \quad\left(z \in \Gamma^{\prime}\right)
$$

et la distance de $\Gamma^{\prime}$ à $l$ est $\geqslant \vartheta=r^{\prime} \sin \vartheta_{0}$.

Les nombres $\varepsilon, \vartheta, N=N_{0}+1$ sont positifs et ne dépendent pas de $z_{0}$; l'énoncé a) du lemme est établi. La démonstration de la partie b) est analogue (on y remplace $\varphi\left(z_{0}, z\right)$ par l'angle de la tangente au point $z$ avec la tangente au point $z_{0}, \theta(z)$ par la distance du point $z$ à la tangente au point $z_{0}$, l'intervalle $\langle 0, \pi / 4\rangle$ par $\left.\left\langle v^{\prime} / 2, v^{\cdot}\right\rangle\right)$.

Convention. Nous diminuons les $L_{j}$ de telle façon que leurs extrínuités soient à une distance $\vartheta$ de $l$ (alors $\Gamma^{\prime}$ augmente).

$\$ 5$. Nous allons maintenant démontrer le résultat principal. Soit, pour $\delta>0$,

$$
\omega(\delta)=\sup _{L \in A,|L| \leqslant \delta} v(L)
$$

le module de continuité de $v$, et $\omega^{-1}$ la fonction inverse. 
THF́nk̀me 5. Soit $\Gamma \in C^{\mathbf{1}}$ une courbe simple (ou un arc simple) d̀ taugente continue, et $\nu \geqslant 0$ une mesure d'énergie finie sur $\Gamma$. Pour toute mesure $\sigma \neq 0$, majorée par $\nu$ et de masse totale 0 :

on a l'inégalité:

$$
|\sigma| \leqslant v, \quad \sigma(\Gamma)=0
$$

$$
\frac{\|\sigma\|^{2}}{[\sigma]^{2}} \geqslant c-\frac{1}{\log \frac{1}{\omega^{-1}(\bar{M}[\bar{\sigma}])}} ;
$$

les constantes positives $c$ et $M<1$ dépendent seulement de $I$.

Démonstration. $1^{\circ}$. Posons $[\sigma]=m$. Nous avons $\sigma \in K(m, v)$. Soient $a, L^{+}, L^{-}$comme dans les théorèmes 1,2 . Il suffit de démontrer

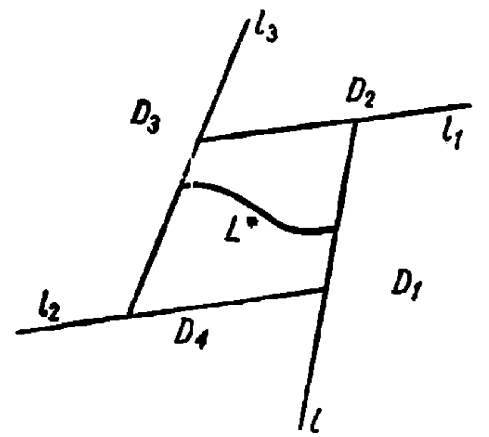

Fig. 3 l'inégalité (15) pour $\sigma=a$. Partageons $L^{+}$ en $n \leqslant N^{*}=|\Gamma| / r$ ares ( $r$ est défini au paragraphe précédent), chacun de longueur $<r$. Sur un de ces arcs - soit $L^{*}$ - on a une masse $\alpha\left(L^{*}\right) \geqslant m / N^{*}$. Soit $z_{0}$ une extrémité de $L^{*} ; l, l_{1}, l$ les droites du lemme 3 ; et $l_{3}$ la droite $l$ pour l'autre extrémité de $L^{*}$ (fig. 3).

$2^{\circ}$. Deux neutralisations des charges nous fourniront les masses positives séparées des charges négatives par une droite satis-

faisant à (14). Soit $Q$ la classe des mesures $\mu$ telles que

$$
\mu(\Gamma)=0, \quad|\mu| \leqslant v, \quad \mu\left(L^{*}\right)=\alpha\left(L^{*}\right)
$$

et $\mu_{0}$ la mesure minimale dans $Q ; \alpha \in Q$, on a donc (1)

$$
\|a\| \geqslant\left\|\mu_{0}\right\|, \quad\left[\mu_{0}\right]=\alpha\left(L^{*}\right), \quad \mu \leqslant 0 .
$$

Soient $D_{1}, \ldots, D_{4}$ les domaines indiqués sur la fig. 3 ; l'un d'eux contient au monis $\frac{1}{4}$ de la masse négative, par exemple $\mu_{0}\left(D_{1}\right) \leqslant-\frac{1}{4}\left[\mu_{0}\right]$. Soit $\Gamma^{-}$la partie de $\Gamma$ séparée de $L^{*}$ par $l \subset \partial D_{1}$ et $\Gamma^{+}=\Gamma-\Gamma^{-}$; on a $\mu_{0}\left(\Gamma^{-}\right)$ $\leqslant-\frac{1}{4}\left[\mu_{0}\right] \leqslant-\frac{1}{4} m / N^{*}$. Soit $P$ la classe des mesures $\tau$, définie par les conditions

$$
|\tau(\Gamma)|=0, \quad|\tau| \leqslant v, \quad \tau\left(\Gamma^{+}\right)=\mu_{n}\left(\Gamma^{+}\right) \geqslant \frac{1}{4} m / N^{*}
$$

et $\tau_{0}$ la mesure minimale dans $P ; \mu_{0} \in P$, d'où (1)

$$
\|\alpha\| \geqslant\left\|\mu_{0}\right\| \geqslant\left\|\tau_{0}\right\|, \quad \tau \geqslant 0 \text { dans } \Gamma^{+}, \quad \tau \leqslant 0 \text { dans } \Gamma^{-} .
$$

(1) L'existence et les propriétés du signe de $\mu_{0}$ et $\tau_{0}$ s'établissent de la même manière que dans $\$ 3$. 
$3^{\circ}$. Prenons $l$ pour axe réel et soit par exemple im $z \geqslant 0$ sur $\Gamma^{+}$. Pour une mesure $\sigma$ quelconque, la mesure antisymétrique $\sigma^{*}$ sera définie par la relation $d \sigma^{*}(z)=-d \sigma(\bar{z})$.

Une mesure sera dite antisymétrique, si $\sigma^{*}=\sigma$. Pour une telle mesure $\sigma^{+*}=-\sigma^{-}$, donc

$$
\begin{aligned}
U^{\sigma}(z) & =\int \log \frac{1}{|z-\zeta|} d\left(\sigma^{+}+\sigma^{+*}\right) \\
& =\int \log \frac{1}{|z-\zeta|} d \sigma^{+}(\zeta)+\int \log \frac{1}{|z-\zeta|}\left(-d \sigma^{+}(\bar{\zeta}) \mid\right. \\
& =\int \log \left|\frac{z-\bar{\zeta}}{z-\zeta}\right| d \sigma^{+}(\zeta),
\end{aligned}
$$

et comme $U^{\sigma}(z)=-U^{\sigma}(\bar{z})$,

$$
\begin{aligned}
\|\sigma\|^{2} & =\int U^{\sigma}(z) d\left(\sigma^{+}+\sigma^{+*}\right)=\int U^{\sigma}(z) d \sigma^{+}(z)+\int U^{\sigma}(\bar{z}) d \sigma^{+}(\bar{z}) \\
& =2 \iint \log \left|\frac{z-\bar{\zeta}}{z-\zeta}\right| d \sigma^{+} d \sigma^{+} \quad\left(\sigma^{*}=\sigma\right) .
\end{aligned}
$$

Nous dirons que deux mesures $\sigma_{1}, \sigma_{2}$ sont cosymétriques, si chacune est antisymétrique et positive dans le demi-plan $\operatorname{im} z \geqslant 0$.

LEMME 4. L'énergie mutuelle de deux mesures cosymétriques est positive: $\left(\sigma_{1}, \sigma_{2}\right) \geqslant 0$.

Démonstration. Le potentiel de $\sigma_{1}$ est positif dans le demi-plan $\operatorname{im} z \geqslant 0$, en particulier sur le support de $\sigma_{2}^{+}$, car $|z-\bar{\zeta}| \geqslant|z-\zeta|$ pour $\operatorname{im} z, \operatorname{im} \zeta \geqslant 0$, et négatif pour $\operatorname{im} z \leqslant 0$, en particulier sur le support de $\sigma_{2}^{-}$; par conséquent

$$
\left(\sigma_{1}, \sigma_{2}\right)=\int U^{\sigma_{1}} d \sigma_{2}^{+}-\int U^{\sigma_{1}} d \sigma_{2}^{-} \geqslant 0 .
$$

$4^{\circ}$. Soit $\tau_{0}^{*}$ la mesure antisymétrique à $\tau_{0}$. Nous avons

$$
\left\|\tau_{0}+\tau_{0}^{*}\right\| \leqslant\left\|\tau_{0}\right\|+\left\|\tau_{0}^{*}\right\|=2\left\|\tau_{0}\right\| \text {. }
$$

Posons

$$
\begin{aligned}
\tau_{j} & =\text { restriction de } \tau_{0} \text { à } L_{j}, \\
\tau^{\prime} & =\text { restriction de } \tau_{0} \grave{a} \Gamma^{\prime}
\end{aligned}
$$

(notations du lemme 3 avec la convention introduite à la fin de sa démonstration) et

On a

$$
\begin{aligned}
\lambda_{0} & =\tau^{\prime}+\tau^{\prime *}, \\
\lambda_{2 j} & =\tau_{j}^{+}+\tau_{j}^{+*}, \\
\lambda_{2 j+1} & =-\tau_{j}^{-}-\tau_{j}^{-*} \quad(j=1, \ldots, n ; n \leqslant N) .
\end{aligned}
$$

$$
\tau_{0}+\tau_{0}^{*}=\sum_{s=0}^{2 n+1} \lambda_{s}, \quad \lambda_{s}=\lambda_{s}^{*},
$$


et par la cosymétrie de toutes les mesures $\lambda_{s}$

$$
\begin{gathered}
4\left\|\tau_{0}\right\|^{2} \geqslant\left\|\tau_{0}+\tau_{0}^{*}\right\|^{2}=\left\|\sum \lambda_{s}\right\|^{2} \geqslant \sum\left\|\lambda_{8}\right\|^{2}, \\
\sum_{s=0}^{2 n+1} \lambda_{s}^{+}(\Gamma) \geqslant m / 2 N^{*} .
\end{gathered}
$$

Done, pour un $8=k, \lambda_{k}^{+}\left(I^{\prime}\right) \geqslant m^{\prime}=m / 2 N^{*}(2 N+2)$. Deux cas sont possibles:

$5^{\circ} k=0$. On a (voir (19), $R=$ diamètre de $\Gamma$ )

$$
\left\|\lambda_{0}\right\|^{2}=2 \iint \log \left|\frac{z-\bar{\zeta}}{z-\zeta}\right| d \lambda_{0}^{+} d \lambda_{0}^{+} \geqslant 2 m^{\prime 2} \log \frac{R+\vartheta}{R} .
$$

Il en résulte l'inégalité (29), d'où nous allons déduire au $\mathrm{n}^{0} 7^{0}$ l'inégalité (15).

$6^{0} k>0$. Posons, pour abréger l'écriture, $\lambda_{k}^{+}=\theta$; par (19) on a

$$
\left\|\lambda_{k}\right\|^{2}=2 \iint \log \left|\frac{z-\bar{\zeta}}{z-\zeta}\right| d \theta d \theta
$$

Le support de $\theta$ est situé sur $A=L_{j} \cap \Gamma^{+}$pour un certain $j$. Cet are est incliné sur l'axe réel $l$ d'un angle $\geqslant \varepsilon$ (voir lemme 3 ). Soit $z_{0}=x_{0}+i \vartheta$

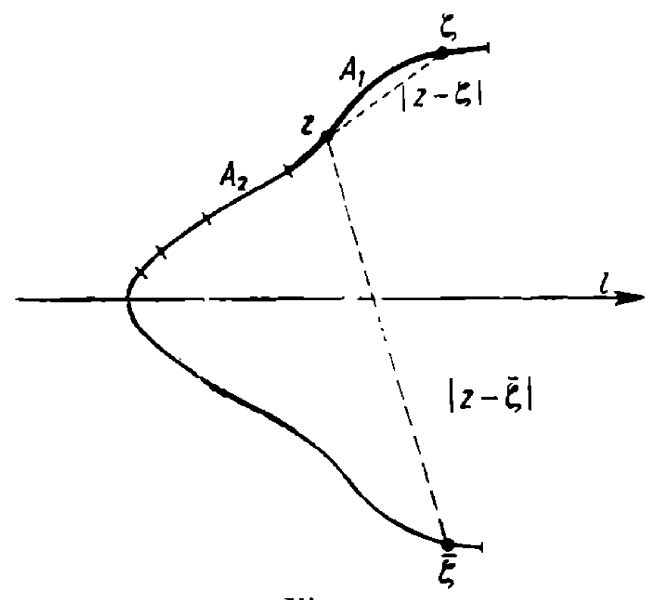

Fig. 4

son extrémité. Partageons $A$ en ares partiels, sur lesquels le noyau de l'intégrale (23) sera borné inférieurement par un nombre positif. Nous posons

(24) $y_{\kappa}=y_{x-1} /(1+\sin \varepsilon)=\vartheta(1+\sin \varepsilon)^{-\varkappa} \quad(\varkappa=0,1,2, \ldots, p), \quad y_{p+1}=0$.

La partie de $A$ où $y_{*} \leqslant \mathrm{imz} \leqslant y_{x-1}$ sera désignée par $A_{x}$; on a (fig. 4) (25) $|z-\bar{\zeta}| /|z-\zeta| \geqslant 2 y_{*} /\left(y_{* \rightarrow 1}-y_{\kappa}\right)(\sin \varepsilon)^{-1}=2 y_{\kappa} / y_{*}=2\left(z, \zeta \in A_{\varkappa}, \varkappa=0, \ldots, p\right)$. 
Soit $\theta_{x}=$ restriction de $\theta$ à $A_{x}(x=1, \ldots, p+1)$. Choisissons $p$ de sorte que l'on ait $\theta_{p+1}(A) \leqslant \frac{1}{2} m^{\prime}$. A cet'effet il suffit que $\omega\left(\left|A_{p+1}\right|\right) \leqslant \frac{1}{2} m^{\prime}$, et nous posons

$$
p=\left[\frac{\log \frac{\vartheta}{\sin \varepsilon} \cdot \frac{1}{\omega^{-1}\left(m^{\prime} / 2\right)}}{\log (1+\sin \varepsilon)}\right]+1 .
$$

Le noyau de nos intégrales étant positif on a par (25), (23)

$$
\begin{aligned}
\frac{1}{2}\left\|\lambda_{k}\right\|^{2} & =\iint \log \left|\frac{z-\bar{\zeta}}{z-\zeta}\right| d\left(\theta_{1}+\ldots+\theta_{p+1}\right) d\left(\theta_{1}+\ldots+\theta_{p+1}\right) \\
& \geqslant \sum_{x=1}^{p} \iint \log \left|\frac{z-\bar{\zeta}}{z-\zeta}\right| d \theta_{x} d \theta_{x} \geqslant \sum_{x=1}^{p}(\log 2) \theta_{x}\left(A_{x}\right)^{2}
\end{aligned}
$$

Mais $\sum_{x=1}^{p} \theta_{x}\left(A_{x}\right) \geqslant \frac{1}{2} m^{\prime}$, donc $\sum_{x=1}^{p} \theta_{x}\left(A_{x}\right)^{2} \geqslant p\left(\frac{1}{2} m^{\prime} / p\right)^{2}$, et nous avons

$$
\left\|\lambda_{k}\right\|^{2} \geqslant(\log 2) m^{\prime 2} / 2 p
$$

$7^{\circ}$ Les inégalités (16), (17), (20)-(22), (26), (28) nous donnent

(29) $\|\alpha\|^{2} \geqslant f_{1}(m) m^{2}, \quad$ où $\quad f_{1}(m)=\frac{1}{32 N^{* 2}(N+1)^{2}} \log \left(1+\frac{\vartheta}{R}\right) \quad$ (cas $5^{\circ}$ ) ou

(30) $\|\alpha\|^{2} \geqslant f_{2}(m) m^{2}, \quad$ où $\quad f_{2}(m)=\frac{2 M^{2} \log (1+\sin \varepsilon)}{\log \left(\frac{\vartheta}{\sin \varepsilon} \cdot \frac{1}{\omega^{-1}(M m)}\right)} \log 2$,

$$
M=\frac{1}{16 N^{*}(N+1)}<1 \quad\left(\operatorname{cas} 6^{\circ}\right) \text {. }
$$

Les paramètres $\vartheta, R, N, N^{*}, \varepsilon, M$ sont positifs et ne dépendent que de $\Gamma$. Posons encore

Les quotients

$$
f(m)=\frac{1}{\log \frac{1}{\omega^{-1}(M m)}}
$$

$$
f(m) / f_{1}(m) \rightarrow 0, \quad f(m) / f_{2}(m) \rightarrow a<\infty \quad(m \rightarrow 0) .
$$

Tenant compte de l'inégalité $\omega^{-1}(M m)<1$, qui résulte de $|\Gamma|<1$, nous en concluons qu'il existe un nombre positif $c$, dépendant seulement de $I$, tel que

$$
f(m) / f_{1}(m) \leqslant 1 / c, \quad f(m) / f_{\mathrm{g}}(m) \leqslant 1 / c \quad(0<m \leqslant v(\Gamma)) .
$$

En rapprochant ces inégalités de (29) et (30), notre théorème se trouvera. démontre - à condition que $U^{v}$ soit continu. 
Nous allons nous débarasser de cette condition $\left({ }^{2}\right)$. Soit $\nu^{*}$ une mesure positive sur $I$ d'énergie finie et $\sigma$ une mesure non nulle de $K\left(m, \nu^{*}\right)$. Il existe alors $([5]$, p. 190$)$ des mesures non nulles $\lambda_{n}^{+}, \lambda_{n}^{-}$de potentiels continus telles que $0 \leqslant \lambda_{n}^{ \pm} \leqslant \sigma^{ \pm},\left\|\sigma^{ \pm}-\lambda_{n}^{ \pm}\right\| \rightarrow 0$. Posons $p_{n}=\lambda_{n}^{+}(\Gamma) / \lambda_{n}^{-}(\Gamma)$ ot $\mu_{n}^{+}=\lambda_{n}^{+}, \mu_{n}^{-}=p_{n} \lambda_{n}^{-}$si $p_{n} \leqslant 1, \mu_{n}^{+}=p_{n}^{-1} \lambda_{n}^{+}, \mu_{n}^{-}=\lambda_{n}^{-}$si $p_{n} \geqslant 1$, enfin $\mu_{n}=\mu_{n}^{+}-$ $-\mu_{n}^{-}$. Puisque $p_{n} \rightarrow 1$, on a $\left\|\mu_{n}^{ \pm}-\sigma^{ \pm}\right\|_{1} \leqslant\left\|\lambda_{n}^{ \pm}-\sigma^{ \pm}\right\|+\left\|\mu_{n}^{ \pm}-\lambda_{n}^{ \pm}\right\| \rightarrow 0$, donc $1^{0}$ $\left\|\mu_{n}\right\| \rightarrow\|\sigma\|, 2^{o}$ par $(1), 0 \leqslant\left(\sigma^{ \pm}-\mu_{n}^{ \pm}\right)(\Gamma) \rightarrow 0$, donc $\left[\mu_{n}\right] \rightarrow[\sigma]$.

Substituons dans l'énoncé du théorème $5, \mu_{n}$ au lieu de $\sigma$, et $\left|\mu_{n}\right|=\mu_{n}^{+}+\mu_{n}^{-}$au lieu de $\nu . U^{v}$ est bien continu, nous avons donc (15):

$$
\left[\mu_{n}\right]^{2} \leqslant e^{-1}\left\|\mu_{n}\right\|^{2} \log 1 / \omega^{-1}\left(M\left[\mu_{n}\right]\right) \leqslant c^{-1}\left\|\mu_{n}\right\|^{2} \log 1 / \omega^{*-1}\left(M\left[\mu_{n}\right]\right),
$$

où $\omega^{*}(\delta)=\sup \left\{\nu^{*}(L),|L| \leqslant \delta, L \in \Lambda\right\}$ est le module de continuite de $\nu^{*}$ et $\omega^{*-1}$ la fonction inverse; $\nu \leqslant \nu^{*}$, donc $\omega \leqslant \omega^{*}$ ce qui entraine la dernière inégalité. Un passage à la limite achève la démonstration $d u$ théorème 5 .

Remarque 2. Nous n'avons pas mentionné la restriction $|\Gamma|<1$ dans l'énoncé du théorème, car elle se lève sans peine. Soit, en effet, $|\Gamma|=l \geqslant 1$. Posons, pour $E \in S, t=1 /(1+l)$

$$
t E=\{\zeta: \zeta=t z, z \in E\} ; \quad \sigma_{t}(t E)=\sigma(E),
$$

$\sigma_{t}$ a son support sur $t \Gamma$, et $\sigma_{t} \in K\left(m, v_{t}\right)$. On a (15) pour $\sigma_{t}$, et comme $($ par $\sigma(\Gamma)=0)\left\|\sigma_{t}\right\|=\|\sigma\|$, pour $\sigma$ aussi. Il n'y a plus qu'd demander explicitement que l'on ait $\omega^{-1}(M m)<1$, c'est-à-dire $m<\omega(1) / M$.

Remarque 3. La condition $\Gamma \in C^{1}$ peut etre affaiblie: il suffit que $\Gamma$ soit la réunion d'un nombre fini d'arcs $\epsilon C^{\mathbf{1}}$ dont aucun n'est tangent à l'autre. Dans ce cas, pourtant, la remarque 1 (§ 3$)$ ne sera pas valable en général.

§6. On peut donner à notre résultat une forme plus expressive. Commençons par le cas particulier où $\nu$ est (ou peut être majorée par) une mesure de densité constante $g$ :

$$
\nu(E) \leqslant g|E| \quad(E \in S),
$$

on, plus généralement, satisfait à la condition de Hölder

$$
\omega(\delta) \leqslant g \delta^{a},
$$

où $a \in(0,1\rangle$ Alor's $\omega^{-1}(\varepsilon) \geqslant(\varepsilon / g)^{1 / a}$ et on a, pour $M<g\left(^{3}\right)$

$$
\frac{\|\sigma\|^{2}}{[\sigma]} \geqslant \frac{c a}{\log \frac{g}{M[\sigma]}}
$$

Par un calcul élémentaire on en tire le

(2) Ajouté au cours de l'impression, ainsi que la modification correspondante dans l'énoncé du théorème 5 .

(') Dans le cas contraire nous diminuons $M$. 
THÉORÈME 6. Soit $v$ une mesure sur $\Gamma, v$ remplissant la condition de Hölder (31) et $\Gamma$ les conditions de la remarque 3 . Il existe un $\Delta=\Delta(\Gamma, g, a)$ $>0$ tel que chaque mesure $\sigma \neq 0$ sur $\Gamma$ pour laquelle

$$
|\sigma| \leqslant \nu, \quad\|\sigma\| \leqslant \Delta, \quad \sigma(\Gamma)=0
$$

satisfait à la condition de Dini-Lipschitz

$$
[\sigma] \leqslant c^{\prime}\|\sigma\| \log \frac{1}{\|\sigma\|} .
$$

Si nous cherchons une condition aussi faible que possible, qui permette de simplifier l'inégalité (15), nous sommes conduits à la condition

$$
\omega(\delta) \leqslant g\left(\log \frac{1}{\delta}\right)^{-b}, \quad b>0 \text {. }
$$

Si $v$ satisfait à cette condition, on obtient une inégalité de Hölder:

$$
[\sigma] \leqslant c^{\prime \prime}\|\sigma\|^{a} \quad \text { où } \quad a=2 b /(2 b+1) \text {. }
$$

Mais, une condition de la forme (34) est toujours remplie. En effet, soit $L$ un arc de longueur $\delta, \nu(L)=\omega(\delta)$, et $\nu_{L}$ la restriction de $\nu$ à $L$. On a, $d(L)$ désignant le diamètre transfini de $L$,

d'où

$$
\|v\|^{2} \geqslant\left\|\nu_{L}\right\|^{2} \geqslant \nu(L)^{2} \log 1 / d(L) \geqslant \omega(\delta)^{2} \log 4 / \delta
$$

$$
\omega(\delta) \leqslant\|\nu\|(\log 1 / \delta)^{-1 / 2},
$$

et nous avons (avec $c^{\prime \prime}=\|\nu\|^{1 / 2} c^{-1 / 4} M^{-1 / 2}$ ) le théorème suivant:

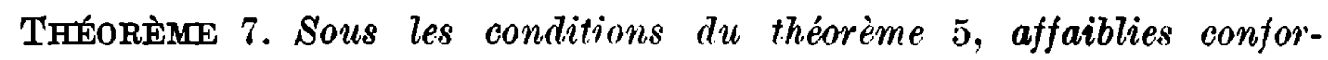
mement à la remarque 2 , on a

$$
[\sigma] \leqslant c^{\prime \prime}\|\sigma\|^{1 / 2}
$$

$\$ 7$. Soit $z=z(t), a \leqslant t \leqslant b$, une représentation de $I$. A l'intervalle $a \leqslant t \leqslant T(T \leqslant b)$ colrespond un arc de $\Gamma$, que nous désignons par $L(z)$ $(z=z(T))$. Posons $s(z)=\sigma(L(z)) ;$ on a $|s(z)| \leqslant[\sigma]$.

Les intégrations par rapport ‘̀ la mesure $\sigma$ et à la fonction $s(z)$ (au sens de Lebesgue-Stjeltjes) étant équivalentes, nous avons pour $z \in \Gamma$

$$
\begin{aligned}
U^{\sigma}(z) & =\int_{\Gamma} \log \frac{1}{|z-\zeta|} d \sigma=\int_{z(a+0)}^{z(b-0)} \log \frac{1}{|z-\zeta|} d s(\zeta) \\
& =\left.s(\zeta) \log \frac{1}{|z-\zeta|}\right|_{i=z(a+0)} ^{z(b-0)}-\int_{\Gamma} s(\zeta) d \log \frac{1}{|z-\zeta|} .
\end{aligned}
$$

Lais $s(z(a+0))=s((b-0))=0$, et la valeur absolue de la dernière intégrale ne surpasse pas $[\sigma]$ Var $\log 1 /|z-\zeta|$. Nous avons ainsi démontré le 
THÉonìme 8. Pour toute mesure $\sigma$ sur $\Gamma$ de masse totale 0

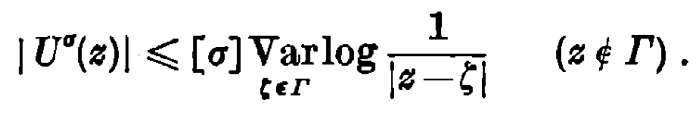

Ce théorème fait ressortir le caractère naturel de la norme [ ]: elle est duale à la variation totale. À l'aide des thaéorèmes 5-7 nous en obtenons deslimitations du potentiel $U^{\sigma}$ par l'énergie de la mesure $\sigma$.

\section{Truvaux cités}

[1] O. Frostmann, Potentiel d'équilibre et capacité des ensembles, Lund 1935.

[2] N. Bourbaki, Éléments de mathématique, VI, Intégration, Paris 1952.

[3] P. Halmos, Measure theory, New York 1950.

[4] G. Anger, Sur le róle des potentiels continus dans les fondements de la théorio du potentiel, Sém. de Théorie du Potentiel, Fac. des Sc. de Paris, $2^{\circ}$ année: 1958.

[5] M. Ohtsuka, On potentiel in locally compact spaoes, J. Sci. Hiroshima Univ. Ser. A-I, 25 (1961), pp. 135.352.

LNSTITUT MATHAMATIQUE DE L'ACADHME POLONAISE DES SCIENCES 